Research Article

\title{
On the Dynamics Behaviors of a Stochastic Echinococcosis Infection Model with Environmental Noise
}

\author{
Jianguo Sun $(\mathbb{D}$, Huina Zhang, and Daqing Jiang $(\mathbb{D}$ \\ School of Science, China University of Petroleum (East China), Qingdao 266555, China \\ Correspondence should be addressed to Daqing Jiang; daqingiiang2010@hotmail.com
}

Received 11 May 2021; Accepted 31 August 2021; Published 6 October 2021

Academic Editor: Mustafa R. S. Kulenovic

Copyright (๑) 2021 Jianguo Sun et al. This is an open access article distributed under the Creative Commons Attribution License, which permits unrestricted use, distribution, and reproduction in any medium, provided the original work is properly cited.

This paper pays main attention to the dynamics behaviors of a stochastic echinococcosis infection model with environmental noise. The existence and uniqueness of the stochastic model is showed in this paper. We obtain the sufficient condition of the ergodic stationary distribution. What is more, the condition of extinction of the stochastic model is also given.

\section{Introduction}

Echinococcosis is a chronic parasitic disease caused by the infection of echinococcosis larvae with human hosts and other mammals [1]. Echinococcus can parasitize many parts of the human body and infect many organs at the same time. At present, the cystic echinococcosis and vesicular echinococcosis are the main types of echinococcosis. Those areas, such as Eastern and Central Asia, Northern Africa, and Southern America, are mainly distributed by echinococcosis [2]. Every year, in the world, there are 18800 new cases of cystic echinococcosis, which causes about 18400 DALYs [3]. Echinococcosis infection is one of the most important public health problems in the world, seriously endangering human health and economic development.

Echinococcosis has a wide range of epidemic and serious harm, and its spread has attracted extensive attention in the world [4-8]. The research on the epidemic area, influencing factors, and transmission mechanism of echinococcosis is a hot topic in recent decades [4-6, 9]. Based on the long-term epidemic situation of echinococcosis, scholars also used a deterministic model to explore the influence of host activity and individual behavior on the transmission of echinococcosis $[9,10]$.

The criteria of global asymptotic stability of the traveling wave solutions of the echinococcosis model were given by $\mathrm{Xu}$ and $\mathrm{Ai}$ [11]. Wang et al. [12] gave the following model of echinococcosis and used the results to Xinjiang of China. The variables in the model are defined in Table 1 , and the parameters are defined in Table 2.

$$
\left\{\begin{array}{l}
\mathrm{d} S_{D}=\left[A_{1}-\beta_{1} S_{D} I_{L}-d_{1} S_{D}+\sigma I_{D}\right] \mathrm{d} t \\
\mathrm{~d} I_{D}=\left[\beta_{1} S_{D} I_{L}-\left(d_{1}+\sigma\right) I_{D}\right] \mathrm{d} t \\
\mathrm{~d} S_{L}=\left[A_{2}-\beta_{2} S_{L} I_{D}-d_{2} S_{L}\right] \mathrm{d} t, \\
\mathrm{~d} I_{L}=\left[\beta_{2} S_{L} I_{D}-d_{2} I_{L}\right] \mathrm{d} t \\
\mathrm{~d} S_{H}=\left[A_{3}-\beta_{3} S_{H} I_{D}-d_{3} S_{H}+\gamma I_{H}\right] \mathrm{d} t \\
\mathrm{~d} E_{H}=\left[\beta_{3} S_{H} I_{D}-\left(d_{3}+\omega\right) E_{H}\right] \mathrm{d} t \\
\mathrm{~d} I_{H}=\left[\omega E_{H}-\left(d_{3}+\mu+\gamma\right) I_{H}\right] \mathrm{d} t
\end{array}\right.
$$

The results showed that the dynamical properties of the model were completely determined by the basic reproduction number $R_{0}\left(R_{0}^{3}=\left(\beta_{1} \beta_{2} A_{1} A_{2} / d_{1} d_{2}^{2}\left(d_{1}+\sigma\right)\right)\right)$. That was, if $R_{0}<1$, the disease-free equilibrium was globally asymptotically stable, and if $R_{0}>1$, the model was permanent and the endemic equilibrium was globally asymptotically stable.

Actually, in the real world, environmental noise is an inevitable important factor, so the epidemic model will be affected by environmental noise [13-20]. In this paper, we will consider a type of environmental noise (namely white noise) with the system (1). Then, the stochastic version corresponding to system (1) can be described by the following equations: 
TABLE 1: Variables in the model (adapted from [12]).

\begin{tabular}{lc}
\hline Population & Definition \\
\hline$S_{D}(t)$ & Susceptible dogs at time $t$ \\
$I_{D}(t)$ & Infectious dogs at time $t$ \\
$S_{L}(t)$ & Susceptible livestock at time $t$ \\
$I_{L}(t)$ & Infectious livestock at time $t$ \\
$S_{H}(t)$ & Susceptible human at time $t$ \\
$E_{H}(t)$ & Exposed human at time $t$ \\
$I_{H}(t)$ & Infectious human at time $t$ \\
\hline
\end{tabular}

Table 2: Parameters in the model (adapted from [12]).

\begin{tabular}{lc}
\hline Parameter & Definition \\
\hline$A_{1}$ & Annual recruitment rate of the dog population \\
$d_{1}$ & Natural death rate of the dog population \\
$\sigma$ & Recovery rate of transition from infected to noninfected dogs \\
$A_{2}$ & Annual recruitment rate of the livestock population \\
$d_{2}$ & Natural death rate of the livestock population \\
$A_{3}$ & Annual recruitment rate of the human population \\
$d_{3}$ & Natural death rate of the human population \\
$\mu$ & Disease-related death rate of the human population \\
$(1 / \omega)$ & Incubation period of infected individuals of the human population \\
$\gamma$ & Recovery rate of the human population \\
$\beta_{1}$ & Infection rate of susceptible dogs by infectious livestock \\
$\beta_{2}$ & Infection rate of susceptible livestock by infectious dogs \\
$\beta_{3}$ & Infection rate of susceptible human by infectious dogs \\
\hline
\end{tabular}

$$
\left\{\begin{array}{l}
\mathrm{d} S_{D}=\left[A_{1}-\beta_{1} S_{D} I_{L}-d_{1} S_{D}+\sigma I_{D}\right] \mathrm{d} t+\sigma_{1} S_{D} \mathrm{~d} B_{1}(t), \\
\mathrm{d} I_{D}=\left[\beta_{1} S_{D} I_{L}-\left(d_{1}+\sigma\right) I_{D}\right] \mathrm{d} t+\sigma_{2} I_{D} \mathrm{~d} B_{2}(t), \\
\mathrm{d} S_{L}=\left[A_{2}-\beta_{2} S_{L} I_{D}-d_{2} S_{L}\right] \mathrm{d} t+\sigma_{3} S_{L} \mathrm{~d} B_{3}(t), \\
\mathrm{d} I_{L}=\left[\beta_{2} S_{L} I_{D}-d_{2} I_{L}\right] \mathrm{d} t+\sigma_{4} I_{L} \mathrm{~d} B_{4}(t), \\
\mathrm{d} S_{H}=\left[A_{3}-\beta_{3} S_{H} I_{D}-d_{3} S_{H}+\gamma I_{H}\right] \mathrm{d} t+\sigma_{5} S_{H} \mathrm{~d} B_{5}(t) \\
\mathrm{d} E_{H}=\left[\beta_{3} S_{H} I_{D}-\left(d_{3}+\omega\right) E_{H}\right] \mathrm{d} t+\sigma_{6} E_{H} \mathrm{~d} B_{6}(t), \\
\mathrm{d} I_{H}=\left[\omega E_{H}-\left(d_{3}+\mu+\gamma\right) I_{H}\right] \mathrm{d} t+\sigma_{7} I_{H} \mathrm{~d} B_{7}(t),
\end{array}\right.
$$

where $B_{i}(t)$ stands for the mutually Brownian motions independently and $B_{i}(0)=0 ; \sigma_{i}^{2}>0$ replaces the intensities of the environmental noise, $i=1,2,3,4,5,6,7$.

In Section 2 of this paper, we give the existence and uniqueness of global positive solutions of the infection model (2) by using the Lyapunov function. The result that the system (2) is ergodic stationary distribution on certain conditions is proved in Section 3. Section 4 gives the sufficient conditions for the existence of extinction of the system (2). We illustrate some simulations to demonstrate our results in Section 5 .

\section{Existence and Uniqueness of the Positive Solution}

Defining a differential operator $L$ associated with equation (1) using the same methods as [15], there is positive and global solution of one system if the coefficients of the system satisfy local Lipschitz conditions. Actually, for the system (2), it may explode in finite time for not satisfying the linear growth condition. Here, we only use the Lyapunov function to proof the solution of the model (2) is positive and global.

Denote

$$
\begin{aligned}
& R_{+}^{n}=\left\{x \in R^{n}: x_{i}>0, \text { for all } 1 \leq i \leq n\right\}, \\
& \bar{R}_{+}^{n}=\left\{x \in R^{n}: x_{i} \geq 0, \text { for all } 1 \leq i \leq n\right\} .
\end{aligned}
$$

We consider the general $\mathrm{n}$-dimensional stochastic differential equation

$$
\mathrm{d} x(t)=f(x(t), t) \mathrm{d} t+g(x(t), t) \mathrm{d} B(t), \quad \text { for } t \geq t_{0},
$$

with initial value $x\left(t_{0}\right)=x_{0} \in R^{n}$, where $B(t)$ denotes $\mathrm{n}$-dimensional standard Brownian motions defined on the above probability space.

Theorem 1. There is a unique solution $\left(S_{D}(t), I_{D}(t)\right.$, $\left.S_{L}(t), I_{L}(t), S_{H}(t), E_{H}(t), I_{H}(t)\right)$ of system (2) on $t \geq 0$ for any initial value $\left(S_{D}(0), I_{D}(0), S_{L}(0), I_{L}(0), S_{H}(0), E_{H}(0)\right.$, $\left.I_{H}(0)\right) \in R_{+}^{7}$, and the solution will remain in $R_{+}^{7}$ with probability 1 , namely, $\left(S_{D}(t), I_{D}(t), S_{L}(t), I_{L}(t), S_{H}(t)\right.$, $\left.E_{H}(t), I_{H}(t)\right) \in R_{+}^{7}$, for all $t \geq 0$ almost surely.

Proof. Using the same method as [17], when the local solution of the system (2) is global with the initial value

$$
\left(S_{D}(0), I_{D}(0), S_{L}(0), I_{L}(0), S_{H}(0), E_{H}(0), I_{H}(0)\right) \in R_{+}^{7},
$$

we only need to prove $\left(S_{D}(t), I_{D}(t), S_{L}(t), I_{L}(t)\right.$, $\left.S_{H}(t), E_{H}(t), I_{H}(t)\right)$ on $t \in\left[0, \tau_{e}\right)$. Let $n_{0} \geq 0$ be a large number and $S_{D}(0), I_{D}(0), S_{L}(0), I_{L}(0), S_{H}(0), E_{H}(0)$, $W(0) \in\left[1 / n_{0}, n_{0}\right]$. Defining the stopping time 


$$
\begin{aligned}
\tau_{n}= & \inf \left\{t \in\left[0, \tau_{e}\right): \min \left\{\left(S_{D}(t), I_{D}(t), S_{L}(t), I_{L}(t), S_{H}(t), E_{H}(t), I_{H}(t)\right)\right\} \leq \frac{1}{n}\right. \\
& \text { or } \left.\max \left\{\left(S_{D}(t), I_{D}(t), S_{L}(t), I_{L}(t), S_{H}(t), E_{H}(t), I_{H}(t)\right)\right\} \geq n\right\},
\end{aligned}
$$

for $n \geq n_{0}$. We set $\inf \phi=\infty$ (as usual $\phi$ denotes the empty set). According to the definition, $\tau_{n}$ is increasing as $n \longrightarrow \infty$. Set $\tau_{\infty}=\lim _{n \longrightarrow \infty} \tau_{n}$, where $\tau_{\infty} \leq \tau_{e}$ a.s. If we can prove that $\tau_{\infty}=\infty$ a.s., then $\tau_{e}=\infty$ and $\left(S_{D}(t), I_{D}(t), S_{L}(t), I_{L}(t), S_{H}(t), E_{H}(t), I_{H}(t)\right) \in R_{+}^{7}$ a.s., for all $t \geq 0$, and the formula $\tau_{\infty}=\infty$ a.s. is needed to show. Otherwise, there exists constants $S>0$ and $\varepsilon \in(0,1)$ such that

$$
P\left\{\tau_{\infty} \leq S\right\}>\varepsilon
$$

Hence, there is an integer $n_{1} \geq n_{0}$ such that

$$
P\left\{\tau_{\infty} \leq S\right\} \geq \varepsilon, \quad \text { for all } n \geq n_{1} .
$$

Define a $C^{2}$-function $V: R_{+}^{7} \longrightarrow \bar{R}_{+}$by

$$
\begin{aligned}
V= & \left(S_{D}-a-a \ln \frac{S_{D}}{a}\right)+\left(I_{D}-b-b \ln \frac{I_{D}}{b}\right)+\left(S_{L}-1-\ln S_{L}\right)+\left(I_{L}-1-\ln I_{L}\right) \\
& +\left(S_{H}-1-\ln S_{H}\right)+\left(E_{H}-1-\ln E_{H}\right)+\left(I_{H}-1-\ln I_{H}\right),
\end{aligned}
$$

where $a, b$ are positive constants to be determined later. The nonnegativity of this function can be seen from $u-1-\log u \geq 0, \forall u>0$. Let $n \geq n_{0}$ and $S>0$ be arbitrary. Using Itô's formula, we obtain

$$
\begin{aligned}
\mathrm{d} V= & L V \mathrm{~d} t+\sigma_{1}\left(S_{D}-a\right) \mathrm{d} B_{1}(t)+\sigma_{2}\left(I_{D}-b\right) \mathrm{d} B_{2}(t)+\sigma_{3}\left(S_{L}-1\right) \mathrm{d} B_{3}(t) \\
& +\sigma_{4}\left(I_{L}-1\right) \mathrm{d} B_{4}(t)+\sigma_{5}\left(S_{H}-1\right) \mathrm{d} B_{5}(t)+\sigma_{6}\left(E_{H}-1\right) \mathrm{d} B_{6}(t)+\sigma_{7}\left(I_{H}-1\right) \mathrm{d} B_{7}(t), \\
L V= & A_{1}-d_{1} S_{D}-a \frac{A_{1}}{S_{D}}+a \beta_{1} I_{L}+a d_{1}-a \sigma \frac{I_{D}}{S_{D}}+\frac{1}{2} a \sigma_{1}^{2} \\
& -b d_{1} I_{D}-b \beta_{1} S_{D} \frac{I_{L}}{I_{D}}+b\left(d_{1}+\sigma+\frac{1}{2} \sigma_{2}^{2}\right)+A_{2}-d_{2} S_{L}-\frac{A_{2}}{S_{L}}+\beta_{2} I_{D}+d_{2}+\frac{1}{2} \sigma_{3}^{2} \\
& -d_{2} I_{L}-\beta_{2} S_{L} \frac{I_{D}}{I_{L}}+d_{2}+\frac{1}{2} \sigma_{4}^{2}+A_{3}-d_{3} S_{H}-\frac{A_{3}}{S_{H}}+\beta_{3} I_{D}+d_{3}-\gamma \frac{I_{H}}{S_{H}}+\frac{1}{2} \sigma_{5}^{2} \\
& -d_{3} E_{H}-\beta_{3} S_{H} \frac{I_{D}}{E_{H}}+d_{3}+\omega+\frac{1}{2} \sigma_{6}^{2}-\left(d_{3}+\mu\right) I_{H}-\omega \frac{E_{H}}{I_{H}}+d_{3}+\mu+\gamma+\frac{1}{2} \sigma_{7}^{2} \\
\leq & \left(a \beta_{1}-d_{2}\right) I_{L}+\left(\beta_{2}+\beta_{3}-b d_{1}\right) I_{D}+A_{1}+A_{2}+A_{3}+\left[(a+b) d_{1}+2 d_{2}+3 d_{3}\right] \\
& +\frac{1}{2}\left(a \sigma_{1}^{2}+b \sigma_{2}^{2}+\sigma_{3}^{2}+\sigma_{4}^{2}+\sigma_{5}^{2}+\sigma_{6}^{2}+\sigma_{7}^{2}\right)+b \sigma+\omega+\mu+\gamma .
\end{aligned}
$$


Choosing then we get

$$
\begin{aligned}
& a=\frac{d_{2}}{\beta_{1}}, \\
& b=\frac{\beta_{2}+\beta_{3}}{d_{1}},
\end{aligned}
$$

$$
\begin{aligned}
L V \leq & A_{1}+A_{2}+A_{3}+\left[(a+b) d_{1}+2 d_{2}+3 d_{3}\right] \\
& +\frac{1}{2}\left(a \sigma_{1}^{2}+b \sigma_{2}^{2}+\sigma_{3}^{2}+\sigma_{4}^{2}+\sigma_{5}^{2}+\sigma_{6}^{2}+\sigma_{7}^{2}\right)+b \sigma+\omega+\mu+\gamma:=K .
\end{aligned}
$$
[14].

The remained proof follows that in Mao et al.'s study

\section{Existence of Unique and Ergodic \\ Stationary Distribution}

Lemma 1 (see [14]). The Markov process $X(t)$ has a stationary distribution $\mu(\cdot)$ if there exists a bounded domain $U \subset E_{l}$ with regular boundary $\Gamma$ and

(i) (B.1) there is a positive number $M$ such that $\sum_{i, j=1}^{l} a_{i j}(x) \xi_{i} \xi_{j} \geq M|\xi|^{2}, x \in U, \xi \in R^{l}$. (ii) (B.2) there exists a nonnegative $C^{2}$ function $V$ such that $L V$ is negative for any $E_{l} \backslash U$. Then,

$$
P_{x}\left\{\lim _{T \longrightarrow \infty} \frac{1}{T} \int_{0}^{T} f(X(t)) \mathrm{d} t=\int_{E_{l}} f(x) \mu(\mathrm{d} x)\right\}=1,
$$

for all $x \in E_{l}$, where $f(\cdot)$ is a function integrable with respect to the measure $\mu$.

Define a parameter

$$
R_{0}^{s}=\frac{\beta_{1} \beta_{2} A_{1} A_{2}}{\left(d_{1}+(1 / 2) \sigma_{1}^{2}\right)\left(d_{1}+\sigma+(1 / 2) \sigma_{2}^{2}\right)\left(d_{2}+(1 / 2) \sigma_{3}^{2}\right)\left(d_{2}+(1 / 2) \sigma_{4}^{2}\right)}
$$

Theorem 2. Assume that $R_{0}^{s}>1$, then there is a unique stationary distribution $\mu(\cdot)$ for system (2) and it has ergodic property.
Proof

(i) Step 1: verify that (B.1) holds. Apparently, the diffusion matrix of system (2) is

$$
\Lambda\left(S_{D}, I_{D}, S_{L}, I_{L}, S_{H}, E_{H}, I_{H}\right)=\left(\begin{array}{ccccccc}
\sigma_{1}^{2} S_{D}^{2} & 0 & 0 & 0 & 0 & 0 & 0 \\
0 & \sigma_{2}^{2} I_{D}^{2} & 0 & 0 & 0 & 0 & 0 \\
0 & 0 & \sigma_{3}^{2} S_{L}^{2} & 0 & 0 & 0 & 0 \\
0 & 0 & 0 & \sigma_{4}^{2} I_{L}^{2} & 0 & 0 & 0 \\
0 & 0 & 0 & 0 & \sigma_{5}^{2} S_{H}^{2} & 0 & 0 \\
0 & 0 & 0 & 0 & 0 & \sigma_{6}^{2} E_{H}^{2} & 0 \\
0 & 0 & 0 & 0 & 0 & 0 & \sigma_{7}^{2} I_{H}^{2}
\end{array}\right) .
$$

Besides, there is $R=\min _{\left(S_{D}, I_{D}, S_{L}, I_{L}, S_{H}, E_{H}, I_{H}\right)} \in R_{+}^{7}$ $\left\{\sigma_{1}^{2} S_{D}^{2}, \sigma_{2}^{2} I_{D}^{2}, \sigma_{3}^{2} S_{L}^{2}, \sigma_{4}^{2} I_{L}^{2}, \sigma_{5}^{2} S_{H}^{2}, \sigma_{6}^{2} E_{H}^{2}, \sigma_{7}^{2} I_{H}^{2}\right\}>0$ such that

$$
\sum_{i, j=1}^{7} \lambda_{i j}\left(S_{D}, I_{D}, S_{L}, I_{L}, S_{H}, E_{H}, I_{H}\right) \xi_{i} \xi_{j}=\sigma_{1}^{2} S_{D}^{2}+\sigma_{2}^{2} I_{D}^{2}+\sigma_{3}^{2} S_{L}^{2}+\sigma_{4}^{2} I_{L}^{2}+\sigma_{5}^{2} S_{H}^{2}+\sigma_{6}^{2} E_{H}^{2}+\sigma_{7}^{2} I_{H}^{2} \geq R|\xi|^{2}
$$


for all $\left(S_{D}, I_{D}, S_{L}, I_{L}, S_{H}, E_{H}, I_{H}\right) \in \bar{U}, \xi \in R^{7}$, which implies condition (B.1) is satisfied.

(ii) Step 2: verify that (B.2) holds. Now, we will construct a nonnegative $C^{2}$-function $V$ and a closed set $U \in \Sigma$ such that

$$
\sup _{\left(S_{D}, I_{D}, S_{L}, I_{L}, S_{H}, E_{H}, I_{H}\right) \in R_{+}^{7} \backslash U} L V\left(S_{D}, I_{D}, S_{L}, I_{L}, S_{H}, E_{H}, I_{H}\right)<0,
$$

which can assure that (B.2) is satisfied. For the convenience of calculation, let

$$
\begin{aligned}
& \bar{S}_{D}=\frac{A_{1}}{d_{1}+(1 / 2) \sigma_{1}^{2}}, \\
& \bar{S}_{L}=\frac{A_{2}}{d_{2}+(1 / 2) \sigma_{3}^{2}} .
\end{aligned}
$$

$\bar{I}_{D}$ and $\bar{I}_{L}$ satisfy the following equations:

$$
\beta_{3} \bar{S}_{L} \bar{I}_{D}=\left(d_{2}+\frac{1}{2} \sigma_{4}^{2}\right) \bar{I}_{L} \text {. }
$$

Beyond that

$$
\begin{gathered}
\widehat{S}_{D}=\frac{S_{D}}{\bar{S}_{D}}, \\
\widehat{I}_{D}=\frac{I_{D}}{\bar{I}_{D}}, \\
\widehat{S}_{L}=\frac{S_{L}}{\bar{S}_{L}} \\
\widehat{I}_{L}=\frac{I_{L}}{\bar{I}_{L}}
\end{gathered}
$$

$$
\begin{aligned}
& \text { Considering a } C^{2} \text {-function } R_{+}^{7} \longrightarrow R \\
& \Phi\left(S_{D}, I_{D}, S_{L}, I_{L}, S_{H}, E_{H}, I_{H}\right)=M V_{1}+V_{2}+V_{3},
\end{aligned}
$$

we assume that $\widetilde{\Phi}$ is the minimum value of $\Phi$. Then, we define a nonnegative $C^{2}$-function $V$,

$$
V=\Phi-\widetilde{\Phi},
$$

where

$$
\begin{aligned}
& V_{1}=-\bar{I}_{D} \ln I_{D}-c_{1} \bar{S}_{D} \ln S_{D}-c_{2} \bar{S}_{L} \ln S_{L}-c_{3} \bar{I}_{L} \ln I_{L}+c I_{L}, \\
& V_{2}=-\ln S_{D}-\ln S_{L}-\ln I_{L}-\ln S_{H}-\ln E_{H}-\ln I_{H}, \\
& V_{3}=\frac{1}{m+2}\left(S_{D}+I_{D}+S_{L}+I_{L}+S_{H}+E_{H}+I_{H}\right)^{m+2}
\end{aligned}
$$

and $c, c_{1}, c_{2}, c_{3}$ are positive constants to be determined later; $m>0$ is a sufficiently small number such that

$$
m<\frac{2\left(d_{1} \wedge d_{2} \wedge d_{3}\right)}{\sigma_{1}^{2} \vee \sigma_{2}^{2} \vee \sigma_{3}^{2} \vee \sigma_{4}^{2} \vee \sigma_{5}^{2} \vee \sigma_{6}^{2} \vee \sigma_{7}^{2}}-1
$$

$M$ is a sufficiently large number such that

$$
A M \bar{I}_{D}+E \leq-2,
$$

where

$$
\begin{aligned}
E= & \sup _{\left(S_{D}, I_{D}, S_{L}, I_{L}, S_{H}, E_{H}, I_{H}\right) \in R_{+}^{7}}\left\{\beta_{1} I_{L}+\left(\beta_{2}+\beta_{3}\right) I_{D}+M c \beta_{2} \varepsilon\left(S_{L}^{m+2}+1\right)\right. \\
& \left.-\frac{1}{2} \rho\left(S_{D}+I_{D}+S_{L}+I_{L}+S_{H}+E_{H}+I_{H}\right)^{m+2}+B+C\right\},
\end{aligned}
$$

where $\varepsilon$ is a sufficiently small number such that

$$
M c \beta_{2} \varepsilon-\frac{1}{2} \rho \leq 0,
$$

and $B, C$ will be defined in (36) and (38), respectively, then

$$
L\left(-\ln S_{D}\right)=-\frac{A_{1}}{S_{D}}+\beta_{1} I_{L}-\sigma \frac{I_{D}}{S_{D}}+d_{1}+\frac{1}{2} \sigma_{1}^{2} .
$$

By applying the inequality $x-1 \geq \ln x(x>0)$, we obtain

$$
L\left(-\bar{S}_{D} \ln S_{D}\right)=-\frac{A_{1}}{\widehat{S}_{D}}+\beta_{1} \bar{S}_{D} I_{L}-\sigma \bar{S}_{D} \frac{I_{D}}{S_{D}}+\bar{S}_{D}\left(d_{1}+\frac{1}{2} \sigma_{1}^{2}\right)
$$

$$
\leq-A_{1}\left(\frac{1}{\widehat{S}_{D}}-1\right)+\beta_{1} \bar{S}_{D} \leq A_{1} \ln \widehat{S}_{D}+\beta_{1} \bar{S}_{D} I_{L} .
$$


From (25), we can obtain

Then,

$$
\frac{\bar{I}_{L}}{\bar{I}_{D}}=\frac{\beta_{2} \bar{S}_{L}}{d_{2}+(1 / 2) \sigma_{4}^{2}} .
$$

$$
\begin{aligned}
& L\left(-\ln I_{D}\right)=-\beta_{1} S_{D} \frac{I_{L}}{I_{D}}+\left(d_{1}+\sigma+\frac{1}{2} \sigma_{2}^{2}\right) \\
& =-\beta_{1} \frac{\bar{S}_{D} \bar{I}_{L}}{\bar{I}_{D}} \frac{\widehat{S}_{D} \widehat{I}_{L}}{\widehat{I}_{D}}+\left(d_{1}+\sigma+\frac{1}{2} \sigma_{2}^{2}\right) \\
& =-\beta_{1} \frac{\bar{S}_{D} \bar{I}_{L}}{\bar{I}_{D}}\left(\frac{\widehat{S}_{D} \widehat{I}_{L}}{\widehat{I}_{D}}-1\right)-\beta_{1} \frac{\bar{S}_{D} \bar{I}_{L}}{\bar{I}_{D}}+\left(d_{1}+\sigma+\frac{1}{2} \sigma_{2}^{2}\right) \\
& \leq-\beta_{1} \bar{S}_{D} \frac{\beta_{2} \bar{S}_{L}}{d_{2}+(1 / 2) \sigma_{4}^{2}}+\left(d_{1}+\sigma+\frac{1}{2} \sigma_{2}^{2}\right)-\beta_{1} \frac{\bar{S}_{D} \bar{I}_{L}}{\bar{I}_{D}} \ln \frac{\widehat{S}_{D} \widehat{I}_{L}}{\widehat{I}_{D}} \\
& =-\left(d_{1}+\sigma+\frac{1}{2} \sigma_{2}^{2}\right)\left(\frac{\beta_{1} \bar{S}_{D} \beta_{2} \bar{S}_{L}}{\left(d_{1}+\sigma+(1 / 2) \sigma_{2}^{2}\right)\left(d_{2}+(1 / 2) \sigma_{4}^{2}\right)}-1\right)-\beta_{1} \frac{\bar{S}_{D} \bar{I}_{L}}{\bar{I}_{D}} \ln \frac{\widehat{S}_{D} \widehat{I}_{L}}{\widehat{I}_{D}} \\
& =-\left(R_{0}^{s}-1\right)\left(d_{1}+\sigma+\frac{1}{2} \sigma_{2}^{2}\right)-\beta_{1} \frac{\bar{S}_{D} \bar{I}_{L}}{\bar{I}_{D}} \ln \frac{\widehat{S}_{D} \widehat{I}_{L}}{\widehat{I}_{D}} \\
& :=-A-\beta_{1} \frac{\bar{S}_{D} \bar{I}_{L}}{\bar{I}_{D}} \ln \frac{\widehat{S}_{D} \widehat{I}_{L}}{\widehat{I}_{D}}, \\
& L\left(-\bar{I}_{D} \ln I_{D}\right) \leq-A \bar{I}_{D}-\beta_{1} \bar{S}_{D} \bar{I}_{L} \ln \frac{\widehat{S}_{D} \widehat{I}_{L}}{\widehat{I}_{D}}, \\
& L\left(-\bar{S}_{L} \ln S_{L}\right)=-\frac{A_{2}}{\widehat{S}_{L}}+\beta_{2} \bar{S}_{L} I_{D}+\bar{S}_{L}\left(d_{2}+\frac{1}{2} \sigma_{3}^{2}\right) \leq A_{2} \ln \widehat{S}_{L}+\beta_{2} \bar{S}_{L} I_{D}, \\
& L\left(-\bar{I}_{L} \ln I_{L}\right)=-\beta_{2} \bar{S}_{L} \bar{I}_{D} \frac{\widehat{S}_{L} \widehat{I}_{D}}{\widehat{I}_{L}}+\bar{I}_{L}\left(d_{2}+\frac{1}{2} \sigma_{4}^{2}\right)=-\beta_{2} \bar{S}_{L} \bar{I}_{D}\left(\frac{\widehat{S}_{L} \widehat{I}_{D}}{\widehat{I}_{L}}-1\right) \\
& \leq-\beta_{2} \bar{S}_{L} \bar{I}_{D} \ln \frac{\widehat{S}_{L} \widehat{I}_{D}}{\widehat{I}_{L}}
\end{aligned}
$$

Then,

$$
\begin{aligned}
L V_{1} \leq & -A \bar{I}_{D}+\left(c_{1} \beta_{1} \bar{S}_{D}-c d_{2}\right) I_{L}+c_{2} \beta_{2} \bar{S}_{L} I_{D}+c \beta_{2} S_{L} I_{D} \\
& +\left(c_{1} A_{1}-\beta_{1} \bar{S}_{D} \bar{I}_{L}\right) \ln \widehat{S}_{D}+\left(\beta_{1} \bar{S}_{D} \bar{I}_{L}-c_{3} \beta_{2} \bar{S}_{L} \bar{I}_{D}\right) \ln \widehat{I}_{D} \\
& +\left(c_{2} A_{2}-c_{3} \beta_{2} \bar{S}_{L} \bar{I}_{D}\right) \ln \widehat{S}_{L}+\left(-\beta_{1} \bar{S}_{D} \bar{I}_{L}+c_{3} \beta_{2} \bar{S}_{L} \bar{I}_{D}\right) \ln \widehat{I}_{L} .
\end{aligned}
$$

Let

$$
\left\{\begin{array}{l}
c_{1} A_{1}-\beta_{1} \bar{S}_{D} \bar{I}_{L}=0 \\
\beta_{1} \bar{S}_{D} \bar{I}_{L}-c_{3} \beta_{2} \bar{S}_{L} \bar{I}_{D}=0, \\
c_{2} A_{2}-c_{3} \beta_{2} \bar{S}_{L} \bar{I}_{D}=0 \\
-\beta_{1} \bar{S}_{D} \bar{I}_{L}+c_{3} \beta_{2} \bar{S}_{L} \bar{I}_{D}=0
\end{array}\right.
$$

then the unique solution of above equations is given as follows:

$$
\begin{gathered}
c_{1}=\frac{\beta_{1} \bar{S}_{D} \bar{I}_{L}}{A_{1}}, \\
c_{2}=\frac{\beta_{1} \bar{S}_{D} \bar{I}_{L}}{A_{2}}, \\
c_{3}=\frac{\beta_{1} \bar{S}_{D} \bar{I}_{L}}{\beta_{2} \bar{S}_{L} \bar{I}_{D}} .
\end{gathered}
$$

Choosing $c=\left(c_{1} \beta_{1} \bar{S}_{D} / d_{2}\right)$, then 


$$
\begin{aligned}
L\left(M V_{1}\right) \leq & -A M \bar{I}_{D}+M c_{2} \beta_{2} \bar{S}_{L} I_{D}+M c \beta_{2} S_{L} I_{D}, \\
L V_{2}= & L\left(-\ln S_{D}-\ln S_{L}-\ln I_{L}-\ln S_{H}-\ln E_{H}-\ln I_{H}\right) \\
= & -\frac{A_{1}}{S_{D}}+\beta_{1} I_{L}-\sigma \frac{I_{D}}{S_{D}}+d_{1}+\frac{1}{2} \sigma_{1}^{2}-\frac{A_{2}}{S_{L}}+\beta_{2} I_{D}+d_{2}+\frac{1}{2} \sigma_{3}^{2}-\beta_{2} S_{L} \frac{I_{D}}{I_{L}}+\left(d_{2}+\frac{1}{2} \sigma_{4}^{2}\right) \\
& -\frac{A_{3}}{S_{H}}+\beta_{3} I_{D}-\gamma \frac{I_{H}}{S_{H}}+d_{3}+\frac{1}{2} \sigma_{5}^{2}-\beta_{3} S_{H} \frac{I_{D}}{E_{H}}+\left(d_{3}+\omega+\frac{1}{2} \sigma_{6}^{2}\right)-\omega \frac{E_{H}}{I_{H}}+\left(d_{3}+\mu+\gamma+\frac{1}{2} \sigma_{7}^{2}\right) \\
\leq & -\frac{A_{1}}{S_{D}}-\frac{A_{2}}{S_{L}}-\beta_{2} S_{L} \frac{I_{D}}{I_{L}}-\frac{A_{3}}{S_{H}}-\beta_{3} S_{H} \frac{I_{D}}{E_{H}}-\omega \frac{E_{H}}{I_{H}}+\beta_{1} I_{L}+\left(\beta_{2}+\beta_{3}\right) I_{D}+B,
\end{aligned}
$$

where

$$
\begin{aligned}
B= & d_{1}+2 \sum_{i=2}^{3} d_{i}+\frac{1}{2}\left(\sigma_{1}^{2}+\sum_{i=3}^{7} \sigma_{i}^{2}\right)+\omega+\mu+\gamma \\
L V_{3}= & L\left(\frac{1}{m+2}\left(S_{D}+I_{D}+S_{L}+I_{L}+S_{H}+E_{H}+I_{H}\right)^{m+2}\right) \\
= & \left(S_{D}+I_{D}+S_{L}+I_{L}+S_{H}+E_{H}+I_{H}\right)^{m+1} \\
& {\left[A_{1}+A_{2}+A_{3}-d_{1}\left(S_{D}+I_{D}\right)-d_{2}\left(S_{L}+I_{L}\right)-d_{3}\left(S_{H}+E_{H}\right)-\left(d_{3}+\mu\right) I_{H}\right] } \\
& +\frac{m+1}{2}\left(S_{D}+I_{D}+S_{L}+I_{L}+S_{H}+E_{H}+I_{H}\right)^{m}\left(\sigma_{1}^{2} S_{D}^{2}+\sigma_{2}^{2} I_{D}^{2}+\sigma_{3}^{2} S_{L}^{2}+\sigma_{4}^{2} I_{L}^{2}+\sigma_{5}^{2} S_{H}^{2}+\sigma_{6}^{2} E_{H}^{2}+\sigma_{7}^{2} I_{H}^{2}\right) \\
\leq & \left(A_{1}+A_{2}+A_{3}\right)\left(S_{D}+I_{D}+S_{L}+I_{L}+S_{H}+E_{H}+I_{H}\right)^{m+1} \\
& -\left(d_{1} \wedge d_{2} \wedge d_{3}\right)\left(S_{D}+I_{D}+S_{L}+I_{L}+S_{H}+E_{H}+I_{H}\right)^{m+2} \\
& +\frac{m+1}{2}\left(\sigma_{1}^{2} \vee \sigma_{2}^{2} \vee \sigma_{3}^{2} \vee \sigma_{4}^{2} \vee \sigma_{5}^{2} \vee \sigma_{6}^{2} \vee \sigma_{7}^{2}\right)\left(S_{D}+I_{D}+S_{L}+I_{L}+S_{H}+E_{H}+I_{H}\right)^{m+2} \\
\leq & C-\frac{1}{2} \rho\left(S_{D}+I_{D}+S_{L}+I_{L}+S_{H}+E_{H}+I_{H}\right)^{m+2},
\end{aligned}
$$

where

$$
\begin{aligned}
C= & \sup _{\left(S_{D}, I_{D}, S_{L}, I_{L}, S_{H}, E_{H}, I_{H}\right) \in R_{+}^{7}}\left\{\left(A_{1}+A_{2}+A_{3}\right)\left(S_{D}+I_{D}+S_{L}+I_{L}+S_{H}+E_{H}+I_{H}\right)^{m+1}\right. \\
& \left.-\frac{1}{2} \rho\left(S_{D}+I_{D}+S_{L}+I_{L}+S_{H}+E_{H}+I_{H}\right)^{m+2}\right\} \\
\rho= & \left(d_{1} \wedge d_{2} \wedge d_{3}\right)-\frac{m+1}{2}\left(\sigma_{1}^{2} \vee \sigma_{2}^{2} \vee \sigma_{3}^{2} \vee \sigma_{4}^{2} \vee \sigma_{5}^{2} \vee \sigma_{6}^{2} \vee \sigma_{7}^{2}\right)>0 .
\end{aligned}
$$

Then, combining with (32), (33), and (35), we get the value of $L V$ finally:

$$
\begin{aligned}
L V \leq & -A M \bar{I}_{D}+c_{2} M \beta_{2} \bar{S}_{L} I_{D}+M c \beta_{2} S_{L} I_{D}-\frac{A_{1}}{S_{D}}-\frac{A_{2}}{S_{L}}-\beta_{2} S_{L} \frac{I_{D}}{I_{L}} \\
& -\frac{A_{3}}{S_{H}}-\beta_{3} S_{H} \frac{I_{D}}{E_{H}}-\omega \frac{E_{H}}{I_{H}}-\frac{1}{2} \rho\left(S_{D}+I_{D}+S_{L}+I_{L}+S_{H}+E_{H}+I_{H}\right)^{m+2} \\
& +\beta_{1} I_{L}+\left(\beta_{2}+\beta_{3}\right) I_{D}+B+C .
\end{aligned}
$$


Then, defining a closed set

$$
\begin{aligned}
U_{\varepsilon} & =\left\{\left(S_{D}, I_{D}, S_{L}, I_{L}, S_{H}, E_{H}, I_{H}\right) \in R_{+}^{7}: \varepsilon \leq S_{D} \leq \frac{1}{\varepsilon}, \varepsilon \leq I_{D} \leq \frac{1}{\varepsilon}, \varepsilon \leq S_{L} \leq \frac{1}{\varepsilon},\right. \\
\varepsilon^{3} & \left.\leq I_{L} \leq \frac{1}{\varepsilon^{3}}, \varepsilon \leq S_{H} \leq \frac{1}{\varepsilon}, \varepsilon^{3} \leq E_{H} \leq \frac{1}{\varepsilon^{3}}, \varepsilon^{4} \leq I_{H} \leq \frac{1}{\varepsilon^{4}}\right\},
\end{aligned}
$$

where $\varepsilon$ is a sufficiently small positive number such that

$$
\begin{gathered}
-\frac{\theta}{\varepsilon}+D \leq-1, \\
-A M \bar{I}_{D}+M c_{2} \beta_{2} \bar{S}_{L} \varepsilon+E \leq-1, \\
-\frac{\theta}{\varepsilon}+F \leq-1,
\end{gathered}
$$

$$
\begin{aligned}
& -\frac{1}{4} \rho \frac{1}{\varepsilon^{m+2}}+G \leq-1, \\
& -\frac{1}{4} \rho \frac{1}{\varepsilon^{3(m+2)}}+G \leq-1, \\
& -\frac{1}{4} \rho \frac{1}{\varepsilon^{4(m+2)}}+G \leq-1,
\end{aligned}
$$

where $\theta=A_{1} \wedge A_{2} \wedge A_{3} \wedge \beta_{2} \wedge \beta_{3} \wedge \omega, D$, E, F are positive constants which can be found from inequations. Divide $R_{+}^{7} U_{\varepsilon}$ to fourteen domains:

$$
\begin{aligned}
U_{1}^{c} & =\left\{\left(S_{D}, I_{D}, S_{L}, I_{L}, S_{H}, E_{H}, I_{H}\right) \in R_{+}^{7}: 0<S_{D}<\varepsilon\right\}, \\
U_{2}^{c} & =\left\{\left(S_{D}, I_{D}, S_{L}, I_{L}, S_{H}, E_{H}, I_{H}\right) \in R_{+}^{7}: 0<I_{D}<\varepsilon\right\}, \\
U_{3}^{c} & =\left\{\left(S_{D}, I_{D}, S_{L}, I_{L}, S_{H}, E_{H}, I_{H}\right) \in R_{+}^{7}: 0<S_{L}<\varepsilon\right\}, \\
U_{4}^{c} & =\left\{\left(S_{D}, I_{D}, S_{L}, I_{L}, S_{H}, E_{H}, I_{H}\right) \in R_{+}^{7}: 0<I_{L}<\varepsilon^{3}, S_{L}>\varepsilon, I_{D}>\varepsilon\right\}, \\
U_{5}^{c} & =\left\{\left(S_{D}, I_{D}, S_{L}, I_{L}, S_{H}, E_{H}, I_{H}\right) \in R_{+}^{7}: 0<S_{H}<\varepsilon\right\}, \\
U_{6}^{c} & =\left\{\left(S_{D}, I_{D}, S_{L}, I_{L}, S_{H}, E_{H}, I_{H}\right) \in R_{+}^{7}: 0<E_{H}<\varepsilon^{3}, S_{H}>\varepsilon, I_{D}>\varepsilon\right\}, \\
U_{7}^{c} & =\left\{\left(S_{D}, I_{D}, S_{L}, I_{L}, S_{H}, E_{H}, I_{H}\right) \in R_{+}^{7}: 0<I_{H}<\varepsilon^{4}, E_{H}>\varepsilon^{3}\right\}, \\
U_{8}^{c} & =\left\{\left(S_{D}, I_{D}, S_{L}, I_{L}, S_{H}, E_{H}, I_{H}\right) \in R_{+}^{7}: S_{D}>\frac{1}{\varepsilon}\right\}, \\
U_{9}^{c} & =\left\{\left(S_{D}, I_{D}, S_{L}, I_{L}, S_{H}, E_{H}, I_{H}\right) \in R_{+}^{7}: I_{D}>\frac{1}{\varepsilon}\right\}, \\
U_{10}^{c} & =\left\{\left(S_{D}, I_{D}, S_{L}, I_{L}, S_{H}, E_{H}, I_{H}\right) \in R_{+}^{7}: S_{L}>\frac{1}{\varepsilon}\right\}, \\
U_{11}^{c} & =\left\{\left(S_{D}, I_{D}, S_{L}, I_{L}, S_{H}, E_{H}, I_{H}\right) \in R_{+}^{7}: I_{L}>\frac{1}{\varepsilon^{3}}\right\}, \\
U_{14}^{c} & =\left\{\left(S_{D}, I_{D}, S_{L}, I_{L}, S_{H}, E_{H}, I_{H}\right) \in R_{+}^{7}: I_{H}>\frac{1}{\varepsilon}\right\}, \\
U_{13}^{c} & =\left\{\left(S_{D}, I_{D}, S_{L}, I_{L}, S_{H}, E_{H}, I_{H}\right) \in R_{+}^{7}: S_{H}>\frac{1}{\varepsilon}\right\}, \\
\left.\left.U_{D}, I_{D}, S_{L}, I_{L}, S_{H}, E_{H}, I_{H}\right) \in R_{+}^{7}: E_{H}>\frac{1}{\varepsilon}\right\}, & \\
\left.U_{1}\right\} &
\end{aligned}
$$


Obviously, $R_{+}^{7} U_{\varepsilon}=\cup_{1 \leq i \leq 14} U_{i}^{c}$. Next, we will show that $L V \leq-1$ on $R_{+}^{7} U_{\varepsilon}$, which is equivalent to verifying it on the above fourteen domains.
Case 1. For any $\left(S_{D}, I_{D}, S_{L}, I_{L}, S_{H}, E_{H}, I_{H}\right) \in U_{1}^{c}$, in view of (39) and (41), thus

$$
\begin{aligned}
L V \leq & -\frac{A_{1}}{S_{D}}-\frac{1}{2} \rho\left(S_{D}+I_{D}+S_{L}+I_{L}+S_{H}+E_{H}+I_{H}\right)^{m+2} \\
& +\beta_{1} I_{L}+\left(M c_{2} \beta_{2} \bar{S}_{L}+\beta_{2}+\beta_{3}\right) I_{D}+M c \beta_{2} S_{L} I_{D}+B+C \\
\leq & -\frac{A_{1}}{\varepsilon}+D \leq-\frac{\theta}{\varepsilon}+D \leq-1,
\end{aligned}
$$

where

$$
\begin{aligned}
D= & \sup _{\left(S_{D}, I_{D}, S_{L}, I_{L}, S_{H}, E_{H}, I_{H}\right) \in R_{+}^{7}}\left\{-\frac{1}{2} \rho\left(S_{D}+I_{D}+S_{L}+I_{L}+S_{H}+E_{H}+I_{H}\right)^{m+2}\right. \\
& \left.+\beta_{1} I_{L}+\left(M c_{2} \beta_{2} \bar{S}_{L}+\beta_{2}+\beta_{3}\right) I_{D}+M c \beta_{2} S_{L} I_{D}+B+C\right\} .
\end{aligned}
$$

Case 2. For any $\left(S_{D}, I_{D}, S_{L}, I_{L}, S_{H}, E_{H}, I_{H}\right) \in U_{2}^{c}$, in view of (24), (39), and (42), thus

$$
\begin{aligned}
L V \leq & -A M \bar{I}_{D}+M c_{2} \beta_{2} \bar{S}_{L} I_{D}+\beta_{1} I_{L}+\left(\beta_{2}+\beta_{3}\right) I_{D}+M c \beta_{2} S_{L} I_{D} \\
& -\frac{1}{2} \rho\left(S_{D}+I_{D}+S_{L}+I_{L}+S_{H}+E_{H}+I_{H}\right)^{m+2}+B+C \\
\leq & -A M \bar{I}_{D}+M c_{2} \beta_{2} \bar{S}_{L} \varepsilon+E \leq-1 .
\end{aligned}
$$

Case 3. For any $\left(S_{D}, I_{D}, S_{L}, I_{L}, S_{H}, E_{H}, I_{H}\right) \in U_{3}^{c}$, in view of

(39) and (41), thus

$$
\begin{aligned}
L V \leq & -\frac{A_{2}}{S_{L}}-\frac{1}{2} \rho\left(S_{D}+I_{D}+S_{L}+I_{L}+S_{H}+E_{H}+I_{H}\right)^{m+2} \\
& +\beta_{1} I_{L}+\left(M c_{2} \beta_{2} \bar{S}_{L}+\beta_{2}+\beta_{3}\right) I_{D}+M c \beta_{2} S_{L} I_{D}+B+C \leq-\frac{A_{2}}{\varepsilon}+D \leq-\frac{\theta}{\varepsilon}+F \leq-1,
\end{aligned}
$$

where

$$
\begin{aligned}
F= & \sup _{\left(S_{D}, I_{D}, S_{L}, I_{L}, S_{H}, E_{H}, I_{H}\right) \in R_{+}^{7}}\left\{\beta_{1} I_{L}+\left(M c_{2} \beta_{2} \bar{S}_{L}+\beta_{2}+\beta_{3}\right) I_{D}+M c \beta_{2} \varepsilon\left(I_{D}^{m+2}+1\right)\right. \\
& \left.-\frac{1}{2} \rho\left(S_{D}+I_{D}+S_{L}+I_{L}+S_{H}+E_{H}+I_{H}\right)^{m+2}+B+C\right\} .
\end{aligned}
$$


Case 4. For any $\left(S_{D}, I_{D}, S_{L}, I_{L}, S_{H}, E_{H}, I_{H}\right) \in U_{4}^{c}$, in view of (39) and (41), thus

$$
\begin{aligned}
L V \leq & -\beta_{2} S_{L} \frac{I_{D}}{I_{L}}-\frac{1}{2} \rho\left(S_{D}+I_{D}+S_{L}+I_{L}+S_{H}+E_{H}+I_{H}\right)^{m+2} \\
& +\beta_{1} I_{L}+\left(M c_{2} \beta_{2} \bar{S}_{L}+\beta_{2}+\beta_{3}\right) I_{D}+M c \beta_{2} S_{L} I_{D}+B+C \\
\leq & -\frac{\beta_{2}}{\varepsilon}+D \leq-\frac{\theta}{\varepsilon}+D \leq-1 .
\end{aligned}
$$

Case 5. For any $\left(S_{D}, I_{D}, S_{L}, I_{L}, S_{H}, E_{H}, I_{H}\right) \in U_{5}^{c}$, in view of (39) and (41), thus

$$
\begin{aligned}
L V \leq & -\frac{A_{3}}{S_{H}}-\frac{1}{2} \rho\left(S_{D}+I_{D}+S_{L}+I_{L}+S_{H}+E_{H}+I_{H}\right)^{m+2} \\
& +\beta_{1} I_{L}+\left(M c_{2} \beta_{2} \bar{S}_{L}+\beta_{2}+\beta_{3}\right) I_{D}+M c \beta_{2} S_{L} I_{D}+B+C \\
\leq & -\frac{A_{3}}{\varepsilon}+D \leq-\frac{\theta}{\varepsilon}+D \leq-1 .
\end{aligned}
$$

Case 6. For any $\left(S_{D}, I_{D}, S_{L}, I_{L}, S_{H}, E_{H}, I_{H}\right) \in U_{6}^{c}$, in view of (39) and (41), thus

$$
\begin{aligned}
L V \leq & -\beta_{3} S_{H} \frac{I_{D}}{E_{H}}-\frac{1}{2} \rho\left(S_{D}+I_{D}+S_{L}+I_{L}+S_{H}+E_{H}+I_{H}\right)^{m+2} \\
& +\beta_{1} I_{L}+\left(M c_{2} \beta_{2} \bar{S}_{L}+\beta_{2}+\beta_{3}\right) I_{D}+M c \beta_{2} S_{L} I_{D}+B+C \\
\leq & -\frac{\beta_{3}}{\varepsilon}+D \leq-\frac{\theta}{\varepsilon}+D \leq-1 .
\end{aligned}
$$

Case 7. For any $\left(S_{D}, I_{D}, S_{L}, I_{L}, S_{H}, E_{H}, I_{H}\right) \in U_{7}^{c}$, in view of (39) and (41), thus

$$
\begin{aligned}
L V \leq & -\omega \frac{E_{H}}{I_{H}}-\frac{1}{2} \rho\left(S_{D}+I_{D}+S_{L}+I_{L}+S_{H}+E_{H}+I_{H}\right)^{m+2} \\
& +\beta_{1} I_{L}+\left(M c_{2} \beta_{2} \bar{S}_{L}+\beta_{2}+\beta_{3}\right) I_{D}+M c \beta_{2} S_{L} I_{D}+B+C \leq-\frac{\omega}{\varepsilon}+D \leq-\frac{\theta}{\varepsilon}+D \leq-1 .
\end{aligned}
$$

Case 8. For any $\left(S_{D}, I_{D}, S_{L}, I_{L}, S_{H}, E_{H}, I_{H}\right) \in U_{8}^{c}$, in view of

(39) and (43), thus

$$
\begin{aligned}
L V \leq & -\frac{1}{4} \rho\left(S_{D}+I_{D}+S_{L}+I_{L}+S_{H}+E_{H}+I_{H}\right)^{m+2} \\
& -\frac{1}{4} \rho\left(S_{D}+I_{D}+S_{L}+I_{L}+S_{H}+E_{H}+I_{H}\right)^{m+2} \\
& +\beta_{1} I_{L}+\left(M c_{2} \beta_{2} \bar{S}_{L}+\beta_{2}+\beta_{3}\right) I_{D}+M c \beta_{2} S_{L} I_{D}+B+C \\
\leq & -\frac{1}{4} \rho\left(S_{D}\right)^{m+2}+G \leq-\frac{1}{4} \rho \frac{1}{\varepsilon^{m+2}}+G \leq-1,
\end{aligned}
$$

where

$$
\begin{aligned}
G= & \sup _{\left(S_{D}, I_{D}, S_{L}, I_{L}, S_{H}, E_{H}, I_{H}\right) \in R_{+}^{7}}\left\{-\frac{1}{4} \rho\left(S_{D}+I_{D}+S_{L}+I_{L}+S_{H}+E_{H}+I_{H}\right)^{m+2}\right. \\
& \left.+\beta_{1} I_{L}+\left(M c_{2} \beta_{2} \bar{S}_{L}+\beta_{2}+\beta_{3}\right) I_{D}+M c \beta_{2} S_{L} I_{D}+B+C\right\} .
\end{aligned}
$$


Case 9. For any $\left(S_{D}, I_{D}, S_{L}, I_{L}, S_{H}, E_{H}, I_{H}\right) \in U_{9}^{c}$, in view of (39) and (43), thus

$$
\begin{aligned}
L V \leq & -\frac{1}{4} \rho\left(S_{D}+I_{D}+S_{L}+I_{L}+S_{H}+E_{H}+I_{H}\right)^{m+2} \\
& -\frac{1}{4} \rho\left(S_{D}+I_{D}+S_{L}+I_{L}+S_{H}+E_{H}+I_{H}\right)^{m+2} \\
& +\beta_{1} I_{L}+\left(M c_{2} \beta_{2} \bar{S}_{L}+\beta_{2}+\beta_{3}\right) I_{D}+M c \beta_{2} S_{L} I_{D}+B+C \leq-\frac{1}{4} \rho\left(I_{D}\right)^{m+2}+G \leq-\frac{1}{4} \rho \frac{1}{\varepsilon} \varepsilon^{m+2}+G \leq-1 .
\end{aligned}
$$

Case 10. For any $\left(S_{D}, I_{D}, S_{L}, I_{L}, S_{H}, E_{H}, I_{H}\right) \in U_{10}^{c}$, in view of (39) and (43), thus

$$
\begin{aligned}
L V \leq & -\frac{1}{4} \rho\left(S_{D}+I_{D}+S_{L}+I_{L}+S_{H}+E_{H}+I_{H}\right)^{m+2}-\frac{1}{4} \rho\left(S_{D}+I_{D}+S_{L}+I_{L}+S_{H}+E_{H}+I_{H}\right)^{m+2} \\
& +\beta_{1} I_{L}+\left(M c_{2} \beta_{2} \bar{S}_{L}+\beta_{2}+\beta_{3}\right) I_{D}+M c \beta_{2} S_{L} I_{D}+B+C \\
\leq & -\frac{1}{4} \rho\left(S_{L}\right)^{m+2}+G \leq-\frac{1}{4} \rho \frac{1}{\varepsilon^{m+2}}+G \leq-1 .
\end{aligned}
$$

Case 11. For any $\left(S_{D}, I_{D}, S_{L}, I_{L}, S_{H}, E_{H}, I_{H}\right) \in U_{11}^{c}$, in view of (39) and (44), thus

$$
\begin{aligned}
L V \leq & -\frac{1}{4} \rho\left(S_{D}+I_{D}+S_{L}+I_{L}+S_{H}+E_{H}+I_{H}\right)^{m+2} \\
& -\frac{1}{4} \rho\left(S_{D}+I_{D}+S_{L}+I_{L}+S_{H}+E_{H}+I_{H}\right)^{m+2} \\
& +\beta_{1} I_{L}+\left(M c_{2} \beta_{2} \bar{S}_{L}+\beta_{2}+\beta_{3}\right) I_{D}+M c \beta_{2} S_{L} I_{D}+B+C \\
\leq & -\frac{1}{4} \rho\left(I_{L}\right)^{m+2}+G \leq-\frac{1}{4} \rho \frac{1}{\varepsilon^{3(m+2)}}+G \leq-1 .
\end{aligned}
$$

Case 12. For any $\left(S_{D}, I_{D}, S_{L}, I_{L}, S_{H}, E_{H}, I_{H}\right) \in U_{12}^{c}$, in view of (39) and (43), thus

$$
\begin{aligned}
L V \leq & -\frac{1}{4} \rho\left(S_{D}+I_{D}+S_{L}+I_{L}+S_{H}+E_{H}+I_{H}\right)^{m+2} \\
& -\frac{1}{4} \rho\left(S_{D}+I_{D}+S_{L}+I_{L}+S_{H}+E_{H}+I_{H}\right)^{m+2} \\
& +\beta_{1} I_{L}+\left(M c_{2} \beta_{2} \bar{S}_{L}+\beta_{2}+\beta_{3}\right) I_{D}+M c \beta_{2} S_{L} I_{D}+B+C \\
\leq & -\frac{1}{4} \rho\left(S_{H}\right)^{m+2}+G \leq-\frac{1}{4} \rho \frac{1}{\varepsilon^{m+2}}+G \leq-1 .
\end{aligned}
$$

Case 13. For any $\left(S_{D}, I_{D}, S_{L}, I_{L}, S_{H}, E_{H}, I_{H}\right) \in U_{13}^{c}$, in view of (39) and (44), thus

$$
\begin{aligned}
L V \leq & -\frac{1}{4} \rho\left(S_{D}+I_{D}+S_{L}+I_{L}+S_{H}+E_{H}+I_{H}\right)^{m+2} \\
& -\frac{1}{4} \rho\left(S_{D}+I_{D}+S_{L}+I_{L}+S_{H}+E_{H}+I_{H}\right)^{m+2} \\
& +\beta_{1} I_{L}+\left(M c_{2} \beta_{2} \bar{S}_{L}+\beta_{2}+\beta_{3}\right) I_{D}+M c \beta_{2} S_{L} I_{D}+B+C \\
\leq & -\frac{1}{4} \rho\left(E_{H}\right)^{m+2}+G \leq-\frac{1}{4} \rho \frac{1}{\varepsilon^{3(m+2)}}+G \leq-1 .
\end{aligned}
$$

Case 14. For any $\left(S_{D}, I_{D}, S_{L}, I_{L}, S_{H}, E_{H}, I_{H}\right) \in U_{14}^{c}$, in view of (39) and (45), thus

$$
\begin{aligned}
L V \leq & -\frac{1}{4} \rho\left(S_{D}+I_{D}+S_{L}+I_{L}+S_{H}+E_{H}+I_{H}\right)^{m+2} \\
& -\frac{1}{4} \rho\left(S_{D}+I_{D}+S_{L}+I_{L}+S_{H}+E_{H}+I_{H}\right)^{m+2} \\
& +\beta_{1} I_{L}+\left(M c_{2} \beta_{2} \bar{S}_{L}+\beta_{2}+\beta_{3}\right) I_{D}+M c \beta_{2} S_{L} I_{D}+B+C \\
\leq & -\frac{1}{4} \rho\left(I_{H}\right)^{m+2}+G \leq-\frac{1}{4} \rho \frac{1}{\varepsilon^{4(m+2)}}+G \leq-1 .
\end{aligned}
$$

Through the discussion of the above ten cases, we can satisfy condition (B.2) of Lemma 1. We complete the proof of Theorem 2. 


\section{Extinction}

Define a parameter

$$
\widehat{R}_{0}=\frac{\beta_{1} S_{D}^{0}+\beta_{2} \int_{0}^{\infty} x \pi(x) \mathrm{d} x+\beta_{3} S_{H}^{0}+\omega}{(1 / 8)\left(\sigma_{2}^{2} \wedge \sigma_{4}^{2} \wedge \sigma_{6}^{2} \wedge \sigma_{7}^{2}\right)}
$$

where

$$
\pi(x)=Q x^{-2-\left(2 d_{2} / \sigma_{3}^{2}\right)} e^{-\left(2 / \sigma_{3}^{2}\right)\left(A_{2} / x\right)}, \quad x \in(0, \infty) .
$$

Theorem 3. Let $\left(S_{D}, I_{D}, S_{L}, I_{L}, S_{H}, E_{H}, I_{H}\right)$ be the solution of system (2) with any initial value

$$
\left(S_{D}(0), I_{D}(0), S_{L}(0), I_{L}(0), S_{H}(0), E_{H}(0), I_{H}(0)\right) \in R_{+}^{7} .
$$

If $\widehat{R}_{0}<1$, then the solution $\left(S_{D}, I_{D}, S_{L}, I_{L}, S_{H}, E_{H}, I_{H}\right)$ of system (2) satisfies

$$
\limsup _{t \longrightarrow \infty} \frac{\ln \left(I_{D}+I_{L}+E_{H}+I_{H}\right)}{t} \leq \beta_{1} S_{D}^{0}+\beta_{2} \int_{0}^{\infty} x \pi(x) \mathrm{d} x+\beta_{3} S_{H}^{0}+\omega-\frac{1}{8}\left(\sigma_{2}^{2} \wedge \sigma_{4}^{2} \wedge \sigma_{6}^{2} \wedge \sigma_{7}^{2}\right)<0 \text { a.s. }
$$

Namely,

$$
\underset{t \longrightarrow \infty}{\limsup } I_{D}=\underset{t \longrightarrow \infty}{\limsup } I_{L}=\underset{t \longrightarrow \infty}{\limsup } E_{H}=\underset{t \longrightarrow \infty}{\limsup } I_{H}=0 .
$$

Also, the distribution of $S_{L}(t)$ converges weakly to the measure which has the density

$$
\pi(x)=Q x^{-2-\left(2 d_{2} / \sigma_{3}^{2}\right)} e^{-\left(2 / \sigma_{3}^{2}\right)\left(A_{2} / x\right)}, \quad x \in(0, \infty),
$$

where $Q$ is a constant such that $\int_{0}^{\infty} \pi(x) \mathrm{d} x=1$.

Proof. Consider the following auxiliary logistic equation with random perturbation

$$
\mathrm{d} X(t)=\left(A_{2}-d_{2} X\right) \mathrm{d} t+\sigma_{3} X \mathrm{~d} B_{3}(t),
$$

with the initial value $X(0)=S_{L}(0)>0$. Setting

$$
\begin{aligned}
& b(x)=A_{2}-d_{2} x, \\
& \sigma(x)=\sigma_{3} x, \quad x \in(0, \infty),
\end{aligned}
$$

we compute that

$\int \frac{b(u)}{\sigma^{2}(u)} \mathrm{d} u=\frac{1}{\sigma_{3}^{2}} \int\left(\frac{A_{2}}{u^{2}}-\frac{d_{2}}{u}\right) \mathrm{d} u=\frac{1}{\sigma_{3}^{2}}\left(-\frac{A_{2}}{x}-d_{2} \ln x\right)+Q$.

Therefore,

$$
e^{\int\left(b(u) / \sigma^{2}(u)\right) \mathrm{d} u}=e^{Q} x^{-\left(d_{2} / \sigma_{3}^{2}\right)} e^{-\left(1 / \sigma_{3}^{2}\right)\left(A_{2} / x\right)} .
$$

Clearly, we have

$$
\int_{0}^{\infty} \frac{1}{\sigma^{2}(x)} e^{\int_{1}^{x}\left(2 b(\tau) / \sigma^{2}(\tau)\right)} \mathrm{d} \tau=C \int_{0}^{\infty} x^{-2-\left(2 d_{2} / \sigma_{3}^{2}\right)} e^{-\left(2 / \sigma_{3}^{2}\right)\left(A_{2} / x\right)} \mathrm{d} x<\infty
$$

Consequently, the condition of Theorem 1.16 in [14] follows clearly from above. Thus, system (72) has the ergodic property, and the invariant density is given by

$$
\pi(x)=Q x^{-2-\left(2 d_{2} / \sigma_{3}^{2}\right)} e^{-\left(2 / \sigma_{3}^{2}\right)\left(A_{2} / x\right)} \mathrm{d} x, \quad x \in(0, \infty),
$$

where $Q$ is a constant such that $\int_{0}^{\infty} \pi(x) \mathrm{d} x=1$. From the ergodic theorem, it follows that

$$
\lim _{t \rightarrow \infty} \frac{1}{t} \int_{0}^{t} x(s) \mathrm{d} s=\int_{0}^{\infty} x \pi(x) \mathrm{d} x \text { a.s. }
$$

Let $X(t)$ be the solution of SDE (72) with the initial value $X(0)=S_{L}(0)>0$, then we can see that

$$
S_{L}(t) \leq X(t) \text { a.s. }
$$

On the other hand, integrating from 0 to $t$ and then dividing by $t$ on both sides of (2) lead to 
TABLE 3: List of parameters.

\begin{tabular}{lccc}
\hline Parameter & Value & Parameter & Value \\
\hline$A_{1}$ & 0.9 & $A_{2}$ & 1.0 \\
$A_{3}$ & 0.7 & $d_{1}$ & 0.02 \\
$d_{2}$ & 0.01 & $d_{3}$ & 0.05 \\
$\beta_{1}$ & 0.08 & $\beta_{2}$ & 0.02 \\
$\beta_{3}$ & 0.08 & $\sigma$ & 0.6 \\
$\mu$ & 0.03 & $\omega$ & 0.3 \\
$\gamma$ & 0.8 & & \\
\hline
\end{tabular}

$$
\left\{\begin{array}{l}
\frac{S_{D}(t)-S_{D}(0)}{t}=A_{1}-\beta_{1}\left\langle S_{D} I_{L}\right\rangle-d_{1}\left\langle S_{D}\right\rangle+\sigma\left\langle I_{D}\right\rangle+\frac{\sigma_{1}}{t} \int_{0}^{t} S_{r} \mathrm{~d} B_{1}(r), \\
\frac{I_{D}(t)-I_{D}(0)}{t}=\beta_{1}\left\langle S_{D} I_{L}\right\rangle-\left(d_{1}+\sigma\right)\left\langle I_{D}\right\rangle+\frac{\sigma_{2}}{t} \int_{0}^{t} I_{D}(r) \mathrm{d} B_{2}(r), \\
\frac{S_{H}(t)-S_{H}(0)}{t}=A_{3}-\beta_{3}\left\langle S_{H} I_{D}\right\rangle-d_{3}\left\langle S_{H}\right\rangle+\gamma\left\langle I_{H}\right\rangle+\frac{\sigma_{5}}{t} \int_{0}^{t} S_{H}(r) \mathrm{d} B_{5}(r), \\
\frac{E_{H}(t)-E_{H}(0)}{t}=\beta_{3}\left\langle S_{H} I_{D}\right\rangle-\left(d_{3}+\omega\right)\left\langle E_{H}\right\rangle+\frac{\sigma_{6}}{t} \int_{0}^{t} E_{H}(r) \mathrm{d} B_{6}(r), \\
\frac{I_{H}(t)-I_{H}(0)}{t}=\omega\left\langle E_{H}\right\rangle-\left(d_{3}+\mu+\gamma\right)\left\langle I_{H}\right\rangle+\frac{\sigma_{7}}{t} \int_{0}^{t} I_{H}(r) \mathrm{d} B_{7}(r) .
\end{array}\right.
$$

We can obtain that then $\frac{S_{D}(t)-S_{D}(0)}{t}+\frac{I_{D}(t)-I_{D}(0)}{t}=A_{1}-d_{1}\left\langle S_{D}\right\rangle_{t}-d_{1}\left\langle I_{D}\right\rangle_{t}$,

$$
\left\langle S_{D}\right\rangle_{t}=\frac{A_{1}-d_{1}\left\langle I_{D}\right\rangle_{t}}{d_{1}}+\varphi_{1}(t)=\frac{A_{1}}{d_{1}}-\left\langle I_{D}\right\rangle_{t}+\varphi_{1}(t)=S_{D}^{0}-\left\langle I_{D}\right\rangle_{t}+\varphi_{1}(t),
$$

where

$$
\begin{aligned}
\varphi_{1}(t)= & -\frac{1}{d_{1}}\left(\frac{S_{D}(t)-S_{D}(0)}{t}+\frac{I_{D}(t)-I_{D}(0)}{t}\right), \\
& \begin{aligned}
\frac{S_{H}(t)-S_{H}(0)}{t}+\frac{E_{H}(t)-E_{H}(0)}{t}+\frac{I_{H}(t)-I_{H}(0)}{t}=A_{3}-d_{3}\left\langle S_{H}\right\rangle_{t}-d_{3}\left\langle E_{H}\right\rangle_{t}-\left(d_{3}+\mu\right)\left\langle I_{H}\right\rangle_{t}, \\
\quad \varphi_{2}(t)=-\frac{1}{d_{3}}\left(\frac{S_{H}(t)-S_{H}(0)}{t}+\frac{E_{H}(t)-E_{H}(0)}{t}+\frac{I_{H}(t)-I_{H}(0)}{t}\right) .
\end{aligned}
\end{aligned}
$$

then

$$
\left\langle S_{H}\right\rangle_{t}=S_{H}^{0}-\left\langle E_{H}\right\rangle_{t}-\frac{d_{3}+\mu}{d_{3}}+\varphi_{2}(t),
$$

From [9], we can obtain that

where

$$
\lim _{t \longrightarrow \infty} \varphi_{i}(t)=0, \quad \text { a.s. } i=1,2 .
$$



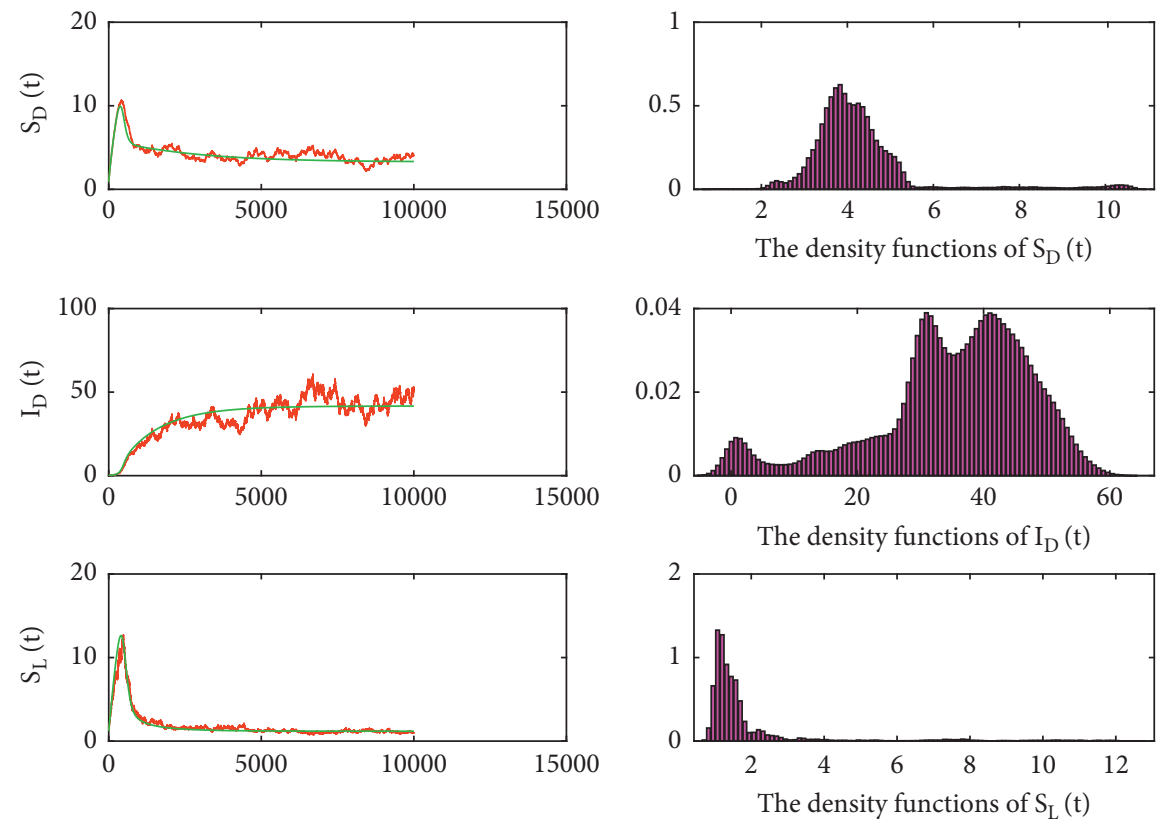

Figure 1: The red lines describe the solution of system (2); the green lines stand for the solution of the corresponding system (1). The right pictures are the histogram of the density function for $S_{D}, I_{D}$, and $S_{L}$ populations.

Let $P(t)=I_{D}+I_{L}+E_{H}+I_{H}$. Applying Itô's formula, we can obtain that

$$
\begin{aligned}
d \ln P(t)= & \left\{\frac{1}{P}\left[\beta_{1} S_{D} I_{L}-\left(d_{1}+\sigma\right) I_{D}+\beta_{2} S_{L} I_{D}-d_{2} I_{L}\right)\right. \\
& \left.+\beta_{3} S_{H} I_{D}-\left(d_{3}+\omega\right) E_{H}+\omega E_{H}-\left(d_{3}+\mu+\gamma\right) I_{H}\right] \\
& \left.-\frac{1}{2 P^{2}}\left(\sigma_{2}^{2} I_{D}^{2}+\sigma_{4}^{2} I_{L}^{2}+\sigma_{6}^{2} E_{H}^{2}+\sigma_{7}^{2} I_{H}^{2}\right)\right\} \mathrm{d} t \\
& +\frac{1}{P}\left[\sigma_{2} I_{D} \mathrm{~d} B_{2}(t)+\sigma_{4} I_{L} \mathrm{~d} B_{4}(t)+\sigma_{6} E_{H} \mathrm{~d} B_{6}(t)+\sigma_{7} I_{H} \mathrm{~d} B_{7}(t)\right] \\
\leq & \left\{\beta_{1} S_{D}+\beta_{2} S_{L}+\beta_{3} S_{H}+\omega-\frac{1}{8}\left(\sigma_{2}^{2} \wedge \sigma_{4}^{2} \wedge \sigma_{6}^{2} \wedge \sigma_{7}^{2}\right)\right\} \mathrm{d} t \\
& +\sigma_{2} \mathrm{~d} B_{2}(t)+\sigma_{4} \mathrm{~d} B_{4}(t)+\sigma_{6} \mathrm{~d} B_{6}(t)+\sigma_{7} \mathrm{~d} B_{7}(t) .
\end{aligned}
$$

Integrating (87) from 0 to $t$ and then dividing by $t$ on both sides, one can see that

$$
\begin{aligned}
& \frac{\ln P(t)}{t}-\frac{\ln P(0)}{t} \leq \beta_{1}\left\langle S_{D}\right\rangle_{t}+\frac{\beta_{2}}{t} \int_{0}^{t} S_{L}(s) \mathrm{d} s+\beta_{3}\left\langle S_{H}\right\rangle_{t} \\
& +\omega-\frac{1}{8}\left(\sigma_{2}^{2} \wedge \sigma_{4}^{2} \wedge \sigma_{6}^{2} \wedge \sigma_{7}^{2}\right)+\frac{\sigma_{2}}{t} B_{2}(t)+\frac{\sigma_{4}}{t} B_{4}(t)+\frac{\sigma_{6}}{t} B_{6}(t)+\frac{\sigma_{7}}{t} B_{7}(t) .
\end{aligned}
$$
has

As application of strong law of large numbers [13], one 

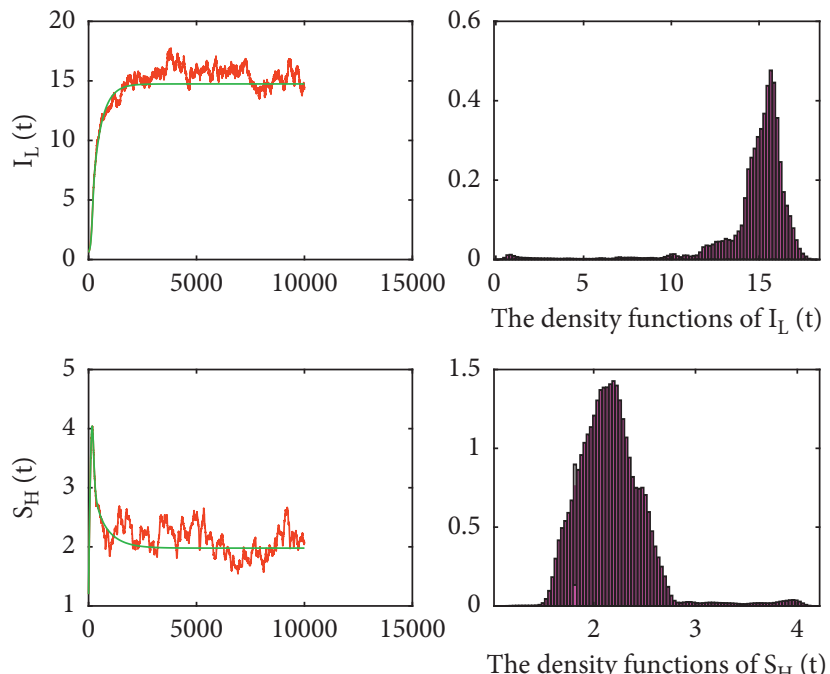

Figure 2: The red lines describe the solution of system (2); the green lines stand for the solution of the corresponding system (1). The right pictures are the histogram of the probability density function for $I_{L}, S_{H}$ populations.
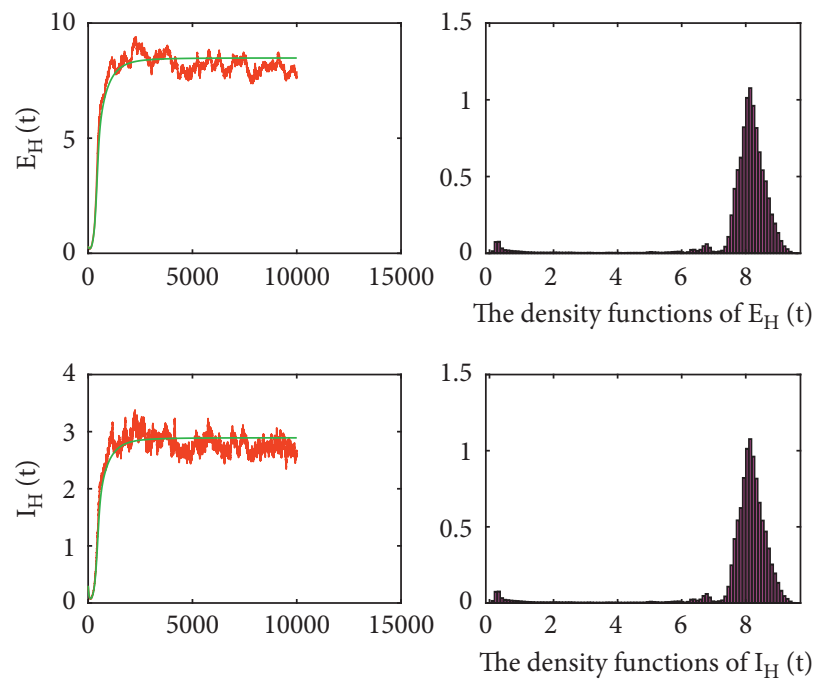

Figure 3: The red lines describe the solution of system (2); the green lines stand for the solution of the corresponding system (1). The right pictures are the histogram of the probability density function for $E_{H}, I_{H}$ populations.

Taking the superior limit on both sides of (88) and note that $\widehat{R}_{0}<1$ leads to

$$
\limsup _{t \longrightarrow \infty} \frac{\ln P(t)}{t} \leq \beta_{1} S_{D}^{0}+\beta_{2} \int_{0}^{\infty} x \pi(x) \mathrm{d} x+\beta_{3} S_{H}^{0}+\omega-\frac{1}{8}\left(\sigma_{2}^{2} \wedge \sigma_{4}^{2} \wedge \sigma_{6}^{2} \wedge \sigma_{7}^{2}\right)<0 \text { a.s }
$$

which implies that

$$
\underset{t \longrightarrow \infty}{\limsup } I_{D}=\limsup _{t \longrightarrow \infty} I_{L}=\limsup _{t \longrightarrow \infty} E_{H}=\limsup _{t \longrightarrow \infty} I_{H}=0 \text { a.s. }
$$

Thus, for any small $\varepsilon>0$, there exist $t_{0}$ and a set $\Omega_{\varepsilon} \subset \Omega$ such that $\mathbf{P}\left(\Omega_{\varepsilon}\right)>1-\varepsilon$ and $\beta_{2} S_{L} I_{D} \leq \varepsilon S_{L}$ for $t \geq t_{0}$ and $\omega \in \Omega_{\varepsilon}$. Now, from 
TABLE 4: List of parameters.

\begin{tabular}{lccc}
\hline Parameter & Value & Parameter & Value \\
\hline$A_{1}$ & 0.5 & $A_{2}$ & 0.6 \\
$A_{3}$ & 0.5 & $d_{1}$ & 0.1 \\
$d_{2}$ & 0.08 & $d_{3}$ & 0.05 \\
$\beta_{1}$ & 0.02 & $\beta_{2}$ & 0.02 \\
$\beta_{3}$ & 0.02 & $\sigma$ & 0.8 \\
$\mu$ & 0.03 & $\omega$ & 0.1 \\
$\gamma$ & 0.8 & & 0 \\
\hline
\end{tabular}

$$
\left[A_{2}-\beta_{2} S_{L} I_{D}-d_{2} S_{L}\right] \mathrm{d} t+\sigma_{3} S_{L} \mathrm{~d} B_{3}(t) \leq \mathrm{d} S_{L}(t) \leq\left[A_{2}-d_{2} S_{L}\right] \mathrm{d} t+\sigma_{3} S_{L} \mathrm{~d} B_{3}(t),
$$

we can obtain that the process $S_{L}(t)$ converges to the measure $\pi$. We finish the proof.

$$
\left(S_{D}(0), I_{D}(0), S_{L}(0), I_{L}(0), S_{H}(0), E_{H}(0), I_{H}(0)\right)=(0.9,0.5,1.2,0.6,1.2,0.3,0.3) .
$$

The corresponding discretizing equations of model (2) are as follows:

$$
\left\{\begin{array}{l}
S_{D}(k+1)=\left[A_{1}-\beta_{1} S_{D}(k) I_{L}(k)-d_{1} S_{D}(k)+\sigma I_{D}(k)\right] \Delta t+\sigma_{1} S_{D}(k) \sqrt{\Delta t} \xi_{1 k}+\frac{\sigma_{1}^{2} S_{D}(k)}{2} \Delta t\left(\xi_{1 k}^{2}-1\right), \\
I_{D}(k+1)=\left[\beta_{1} S_{D}(k) I_{L}(k)-\left(d_{1}+\sigma\right) I_{D}(k)\right] \Delta t+\sigma_{2} I_{D}(k) \sqrt{\Delta t} \xi_{2 k}+\frac{\sigma_{2}^{2} I_{D}(k)}{2} \Delta t\left(\xi_{2 k}^{2}-1\right), \\
S_{L}(k+1)=\left[A_{2}-\beta_{2} S_{L}(k) I_{D}(k)-d_{2} S_{L}(k)\right] \Delta t+\sigma_{3} S_{L}(k) \sqrt{\Delta t} \xi_{3 k}+\frac{\sigma_{3}^{2} S_{L}(k)}{2} \Delta t\left(\xi_{3 k}^{2}-1\right), \\
I_{L}(k+1)=\left[\beta_{2} S_{L}(k) I_{D}(k)-d_{2} I_{L}(k)\right] \Delta t+\sigma_{4} I_{L}(k) \sqrt{\Delta t} \xi_{4 k}+\frac{\sigma_{4}^{2} I_{L}(k)}{2} \Delta t\left(\xi_{4 k}^{2}-1\right), \\
S_{H}(k+1)=\left[A_{3}-\beta_{3} S_{H}(k) I_{D}(k)-d_{3} S_{H}(k)+\gamma I_{H}(k)\right] \Delta t+\sigma_{5} S_{H}(k) \sqrt{\Delta t} \xi_{5 k}+\frac{\sigma_{5}^{2} S_{H}(k)}{2} \Delta t\left(\xi_{5 k}^{2}-1\right), \\
E_{H}(k+1)=\left[\beta_{3} S_{H}(k) I_{D}(k)-\left(d_{3}+\omega\right) E_{H}(k)\right] \Delta t+\sigma_{6} E_{H}(k) \sqrt{\Delta t} \xi_{6 k}+\frac{\sigma_{6}^{2} E_{H}(k)}{2} \Delta t\left(\xi_{6 k}^{2}-1\right), \\
I_{H}(k+1)=\left[\omega E_{H}(k)-\left(d_{3}+\mu+\gamma\right) I_{H}(k)\right] \Delta t+\sigma_{7} I_{H}(k) \sqrt{\Delta t} \xi_{7 k}+\frac{\sigma_{7}^{2} I_{H}(k)}{2} \Delta t\left(\xi_{7 k}^{2}-1\right),
\end{array}\right.
$$

where the time increment $\Delta t$ is positive and $\xi_{i k}$ are the Gaussian random variables which follow the distribution $N(0,1), i=1,2,3,4,5,6,7$.
Example 1. Firstly, we choose the values of system parameters to test the existence of ergodic stationary distribution of the system (94) $\sigma_{1}^{2}=10^{-4}, \sigma_{2}^{2}=3.6 \times 10^{-3}$, 


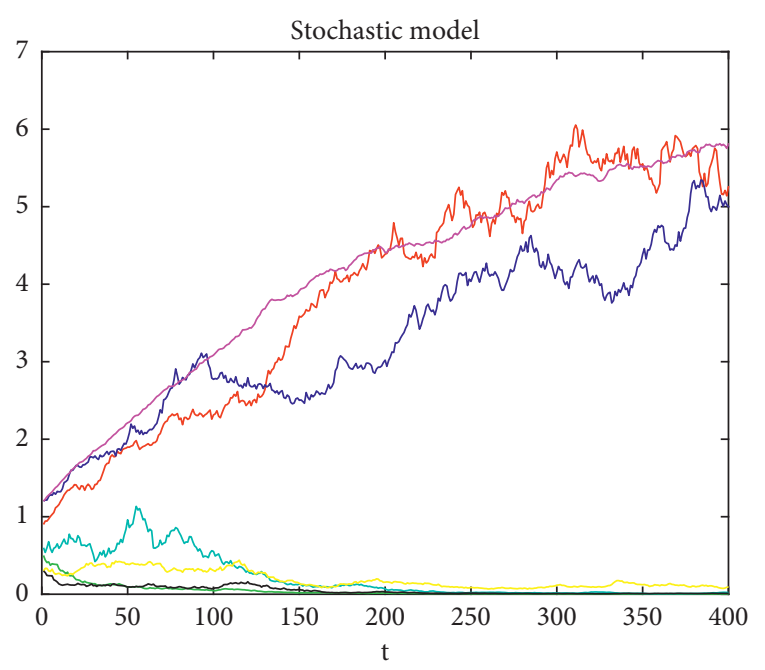

Figure 4: The red line, blue line, and magenta line represent $S_{D}, S_{L}$, and $S_{H}$, respectively; the green line, cyan line, yellow line, and black line represent $I_{D}, I_{L}, E_{H}$, and $I_{H}$, respectively.

$\sigma_{3}^{2}=10^{-2}, \sigma_{4}^{2}=4 \times 10^{-4}, \sigma_{5}^{2}=10^{-4}, \quad \sigma_{6}^{2}=4 \times 10^{-4}, \sigma_{7}^{2}=$ $2.5 \times 10^{-3}$. For other values of the system parameters, see Table 3. Direct calculation leads to $R_{0}^{s}=754.928752>1$, where $R_{0}^{s}$ is defined before Theorem 2 . In other words, the conditions of Theorem 2 hold. In view of Theorem 2, there is an ergodic stationary distribution $\mu(\cdot)$ of system (2). Figures 1-3 illustrate this.

Example 2. In order to obtain the extinction of the infection, in (2), we choose the values of the system parameters as follows: $\sigma_{1}^{2}=10^{-2}, \sigma_{2}^{2}=9 \times 10^{-2}, \sigma_{3}^{2}=10^{-2}, \sigma_{4}^{2}=1.6 \times 10^{-1}$, $\sigma_{5}^{2}=4 \times 10^{-4}, \sigma_{6}^{2}=9 \times 10^{-2} ; \sigma_{7}^{2}=1.6 \times 10^{-1}$. For other values of the system parameters, see Table 4 . By Theorem 3 , $\left(I_{D}, I_{L}, E_{H}, I_{H}\right)$ will tend to be zero exponentially with probability one. We give the simulations to support our results in Figure 4.

\section{Data Availability}

All data in this article are accessible.

\section{Conflicts of Interest}

The authors declare that they have no conflicts of interest.

\section{Authors' Contributions}

All authors carried out the proof and conceived the study. All authors read and approved the final manuscript.

\section{Acknowledgments}

This work was supported by the National Natural Science Foundation of China (No. 11871473) and Shandong Provincial Natural Science Foundation (No. ZR2019MA010).

\section{References}

[1] R. K. Davidson, T. Romig, E. Jenkins, M. Tryland, and L. J. Robertson, "The impact of globalisation on the distribution of Echinococcus multilocularis," Trends in Parasitology, vol. 28, no. 6, pp. 239-247, 2012.

[2] H. Wen, L. Vuitton, T. Tuxun et al., "Echinococcosis: advances in the 21st century," Clinical Microbiology Reviews, vol. 32, no. 2, pp. e00075-18, 2019.

[3] P. R. Torgerson, B. Devleesschauwer, N. Praet et al., "World health organization estimates of the global and regional disease burden of 11 foodborne parasitic diseases, 2010: a data synthesis," PLoS Medicine, vol. 12, no. 12, Article ID e1001920, 2015.

[4] Y. F. Qi, W. P. Wu, and S. C. Li, "Analysis of the epidemic situation of echinococcosis in aba prefecture, sichuan province," The Journal of Pathology, vol. 6, pp. 541-543, 2016.

[5] S. Lahmar, H. Debbek, L. H. Zhang et al., "Transmission dynamics of the Echinococcus granulosus sheep-dog strain (G1 genotype) in camels in Tunisia," Veterinary Parasitology, vol. 121, no. 1-2, pp. 151-156, 2004.

[6] L. Wu, B. Song, W. Dan, and J. Lou, "Mathematical modelling and control of echinococcus in Qinghai province, China," Mathematical Biosciences and Engineering, vol. 10, no. 2, pp. 425-444, 2013.

[7] P. R. Torgerson, K. K. Burtisurnov, B. S. Shaikenov, A. T. Rysmukhambetova, A. M. Abdybekova, and A. E. Ussenbayev, "Modeling the transmission dynamics of Echinococcus granulosus in sheep and cattle in Kazakhstan," Veterinary Parasitology, vol. 114, no. 3, pp. 143-153, 2003.

[8] K. Wang, Z. Teng, and X. Zhang, "Dynamical behaviors of an Echinococcosis epidemic model with distributed delays," Mathematical Biosciences and Engineering: MBE, vol. 14, no. 5-6, pp. 1425-1445, 2017.

[9] G. Zhu, S. Chen, B. Shi, H. Qiu, and S. Xia, "Dynamics of echinococcosis transmission among multiple species and a case study in Xinjiang, China," Chaos, Solitons \& Fractals, vol. 127, pp. 103-109, 2019.

[10] R. Budgey, J. Learmount, and G. C. Smith, "Simulating control of a focal wildlife outbreak of Echinococcus multilocularis," Veterinary Parasitology, vol. 237, pp. 47-56, 2017.

[11] Z. Xu and C. Ai, "A spatial echinococcosis transmission model with time delays: stability and traveling waves," International Journal of Biomathematics, vol. 10, no. 6, pp. 1-32, 2017.

[12] K. Wang, X. Zhang, Z. Jin, H. Ma, Z. Teng, and L. Wang, "Modeling and analysis of the transmission of echinococcosis with application to Xinjiang uygur autonomous region of China," Journal of Theoretical Biology, vol. 333, no. 52, pp. 78-90, 2013.

[13] F. Li, S. Zhang, and X. Meng, "Dynamics analysis and numerical simulations of a delayed stochastic epidemic model subject to a general response function," Computational and Applied Mathematics, vol. 38, no. 95, pp. 1-30, 2019.

[14] X. Mao, G. Marion, and E. Renshaw, "Environmental Brownian noise suppresses explosions in population dynamics," Stochastic Processes and Their Applications, vol. 97, no. 1, pp. 95-110, 2002.

[15] X. Mao, Stochastic Differential Equations and Applications, Horwood, Chichester, UK, 1997.

[16] D. J. Higham, "An algorithmic introduction to numerical simulation of stochastic differential equations," SIAM Review, vol. 43, no. 3, pp. 525-546, 2001.

[17] K. Qi and D. Jiang, "Threshold behavior in a stochastic HTLVI infection model with CTL immune response and regime 
switching," Mathematical Methods in the Applied Sciences, vol. 41, no. 16, pp. 6866-6882, 2018.

[18] B. Zhou, X. Zhang, and D. Jiang, "Dynamics and density function analysis of a stochastic SVI epidemic model with half saturated incidence rate," Chaos, Solitons \& Fractals, vol. 137, p. $109865,2020$.

[19] G. Liu, H. Qi, Z. Chang, and X. Meng, "Asymptotic stability of a stochastic May mutualism system," Computers \& Mathematics with Applications, vol. 79, no. 3, pp. 735-745, 2020.

[20] H. Qi, X. Leng, X. Meng, and T. Zhang, "Periodic solution and ergodic stationary distribution of SEIS dynamical systems with active and latent patients," Qualitative Theory of Dynamical Systems, vol. 18, no. 2, pp. 347-369, 2019. 